\title{
Propuesta de un índice de bienestar social en los países menos adelantados (PMA) de África
}

\section{Proposition of a wellbeing index in the least advanced countries (LAC) of Africa}

\author{
José Antonio Rodríguez-Martín*
}

\begin{abstract}
In this article we present a new measurement proposal applied to a group of countries specially affected by poverty (Least Advanced Countries, LAC), limited by a territory which gives them homogeneity, such as Africa. Our index was constructed paying attention to a larger number of variables than the most used wellbeing indexes, and according to the criteria defined in the objectives of the Millennium Declaration. To sum up, the objective is to produce a global indicator of the fulfillment of these goals; in views of this, we use the $P_{2}$ distance method for the year 2006, which is the most recent year we found with definite data. This index integrates socioeconomic variables that allow a territorial ordering of LAC in Africa in function of these partial indicators.
\end{abstract}

Keywords: synthetic indicators, territorial disparities, wellbeing, least advanced countries, African economies, $P_{2}$ distance measure.

\section{Resumen}

En este artículo se presenta una nueva propuesta de medición aplicada a un grupo de países especialmente afectados por la pobreza (países menos adelantados, PMA), delimitados en un territorio que le dé homogeneidad, como África. Nuestro índice se construyó atendiendo a un número de variables más amplia que los índices de bienestar más utilizados y de acuerdo con los criterios definidos en los objetivos de la Declaración del Milenio. En definitiva, el objetivo es elaborar un indicador global de cumplimiento de estas metas. Para este propósito se emplea el método de distancia $\mathrm{P}_{2}$ para el año 2006, que es el más reciente que encontramos con datos definitivos. Este índice integra variables socioeconómicas que permiten una ordenación territorial de los PMA de África, en función de esos indicadores parciales.

Palabras clave: indicadores sintéticos, disparidades territoriales, bienestar, países menos adelantados, economías africanas, medida de distancia $\mathrm{P}_{2}$.

\footnotetext{
*Universidad de Granada, Espańa. Correo-e: josearm@ugr.es.
} 


\section{Introducción}

El propósito de la economía del bienestar es explicar cómo se puede identificar y alcanzar una asignación de recursos socialmente eficiente (Sánchez y Rodríguez, 2003: 100). En este sentido, según el Instituto Nacional de Estadística de España (INE, 1999), sólo será posible aproximarse a esta realidad mediante el estudio de las condiciones de vida de la población, a través de distintos aspectos sociales: población, familia, educación, trabajo, renta, distribución y consumo, protección social, salud, entorno físico, cultura y ocio, y cohesión y participación social.

Amartya Sen planteó la relevancia de otras dimensiones en el concepto del bienestar, entre las que se pueden citar la salud, la educación, etc. (Sen, 1991; Jasek-Rysdahl, 2001). De acuerdo con los últimos conceptos se configuró la medición del índice que elabora el Programa de Naciones Unidas para el Desarrollo (PNUD). ${ }^{1}$

Con el objetivo de aproximarnos al nivel de bienestar social de los países menos adelantados (PMA) de África, nos inclinamos por el enfoque de los indicadores sociales. La metodología que se emplea se basa en la construcción de un índice sintético mediante la distancia $\mathrm{P}_{2}$ de Pena Trapero, en función de un conjunto de variables intermedias, que contribuyen a cuantificar algún aspecto del concepto que se quiere sintetizar. En nuestro caso, se pretende estudiar el cumplimiento de los Objetivos de Desarrollo del Milenio de la onu, así como analizar la repercusión que tiene cada indicador social respecto a los demás en la determinación de los resultados finales.

En este sentido, los indicadores sintéticos, como el que aplicamos en nuestro trabajo, integran la información de las variables que guardan relación con el nivel de bienestar, el desarrollo sostenible u otra variable de una sociedad, con una perspectiva multidimensional, como el enfoque adoptado por los diversos estudios sobre pobreza del Banco Mundial (2001).

La elección de los países se hizo en función de la lista de los PMA de África que elaboró el Comité de Políticas de Desarrollo (CPD) en 2006, entre los cuales se encuentran territorios pobres y vulnerables a la crisis económica mundial, lo que puede poner en peligro los progresos logrados en avanzar en los Objetivos de Desarrollo del Milenio (ODM), en el horizonte temporal del año 2015.

En particular, el indicador de la ONU para elaborar la lista de los PMA, que incluye los países de África analizados en nuestro trabajo, se basa en

${ }^{1}$ Véase, PNUd (varios años); Chasco y Hernández (2003). 
los siguientes criterios: ${ }^{2}$ el promedio estimado de un periodo de tres ańos de los ingresos nacionales brutos per cápita; la debilidad de recursos humanos, que se relaciona con un índice combinado de capital humano y en un índice de la vulnerabilidad económica.

En esta línea, en estudios futuros pretendemos averiguar si posibles divisiones en la actual agrupación geográfica de los PMA de África arrojarían algunas diferencias relevantes en los valores del índice respecto al caso del presente trabajo, en el que se consideran todos los PMA del continente en conjunto.

Con estos objetivos, primero, se repasa el concepto de bienestar social y distintos enfoques que se pueden seguir para su medición, recogiendo las propuestas metodológicas formuladas por organizaciones e instituciones internacionales. Segundo, se expone la metodología del indicador sintético $\mathrm{DP}_{2} \mathrm{y}$ sus principales propiedades matemáticas y ventajas frente a la utilización de otros procedimientos. Tercero, con el fin de disponer de un instrumento que nos permita evaluar el grado de cumplimiento de los Objetivos del Milenio de la onu para el caso de los PMA de África, se propone construir un indicador global de avances de los países en esta dirección, para este propósito se empleará el método de distancia $\mathrm{P}_{2}$. Finalmente, se presentan los resultados obtenidos y las principales conclusiones que se desprenden.

\section{El concepto del bienestar social y su medición: el enfoque de los indicadores sociales}

El desarrollo económico y su efecto en el bienestar social de los países ha sido objeto de numerosos estudios en la ciencia económica, donde destacan los trabajos de Pigou (1920), Edgeworth (1897), Pareto (1906), Hicks (1939), entre otros muchos. La medición del nivel de bienestar, además de establecer una forma nueva de medir las condiciones de vida de los países, también permite realizar comparaciones entre diferentes territorios.

2 a) Un ingreso bajo, medido por el ingreso nacional bruto (INB) per cápita (promedio de tres años, 2002-2004), con umbrales de 750 dólares para incluir países en la lista y de 900 dólares para excluirlos; b) las existencias de activos humanos, medidas por un índice compuesto (el de activos humanos) basado en indicadores de: 1) nutrición (porcentaje de la población desnutrida), 2) salud (tasa de mortalidad infantil), 3) escolarización (tasa bruta de escolarización secundaria), y 4) alfabetización (tasa de alfabetización de adultos); y c) La vulnerabilidad económica, medida por un índice compuesto (de vulnerabilidad económica) basado en indicadores de: 1) choques naturales (índice de inestabilidad de la producción agrícola; porcentaje de la población desplazada por desastres naturales), 2) choques comerciales (índice de inestabilidad de las exportaciones de bienes y servicios), 3) vulnerabilidad a los choques (parte del PIB correspondiente a la agricultura, la silvicultura y la pesca, índice de concentración de las exportaciones de mercancías), 4) tamaño pequeño de la economía (población expresada en logaritmos), y 5) lejanía (índice de lejanía). 
El enfoque de los indicadores sociales representó un nuevo planteamiento que rompía la fuerte relación entre el crecimiento económico y los principales componentes del bienestar en los países más adelantados (García y Puig, 1980), dotando a este concepto de una mayor amplitud que la del simple crecimiento o desarrollo económico, de manera que éstos son necesarios para el bienestar, pero no suficientes. De acuerdo con el INE, el bienestar social consiste en la "igualdad de oportunidades extendida a todos los bienes, materiales e inmateriales, que se consideran socialmente deseables" (1999: 13).

Por su parte, la Organización para la Cooperación y el Desarrollo Económico (OCDE) define un indicador social como "una medida directa y válida que revela niveles y cambios sobre el tiempo" (2002: 27), que no sólo debe cubrir el conjunto de las preocupaciones sociales fundamentales, sino también tiene que ser más que una descripción estrecha de un fenómeno social.

La situación de pobreza y falta de bienestar de los ciudadanos de un territorio se refiere a su nivel de ingresos, pero también a otros factores como la salud, el empleo, las condiciones de su vivienda, etc. (Banco Mundial, 2001), en el marco de una visión actual más amplia del bienestar, que supera la tradicional identidad del desarrollo o bienestar con la producción o la renta per cápita (Sen, 1999). En este sentido, para el Sistema Europeo de Medición del Bienestar (Consejo de Europa, 2003), éste comprende dos distintas aproximaciones teóricas que apuntan a la conceptualización y medición final del bienestar: los conceptos de calidad de vida y calidad de las sociedades (este último engloba los enfoques de: habitabilidad de las naciones; cohesión, exclusión y el capital social; sustentabilidad; desarrollo humano, y calidad social). En cualquier caso, los indicadores de bienestar dependen, en gran medida, de lo que se pretende conseguir con su utilización, de ahí que existan diversas definiciones.

Una metodología muy aplicada es la propuesta por la ONU. En 1960, el Consejo Económico y Social de las Naciones Unidas, en el informe Definición Internacional y Medida de los Niveles de Vida, propuso determinar los componentes del nivel de vida para elaborar a continuación los indicadores o medidas estadísticas dentro de cada componente. Posteriormente se fue perfeccionado el método, se ampliaron los componentes del bienestar y se mejoró la calidad de los indicadores.

Por su parte, el Sistema de Estadísticas Sociales y Demográficas de la onU define los indicadores sociales como series resumidas relativas al estado y las tendencias de las condiciones de vida, así como la disponibilidad y desempeño de los servicios sociales conexos.

En particular, uno de los indicadores con mayor repercusión en la actualidad, el índice de desarrollo humano (IDH) es una medición por 
país, elaborada por el Programa de las Naciones Unidas para el Desarrollo (PNUD), que se define como "el proceso de ampliar las oportunidades de los individuos para que el desarrollo en los países sea democrático y participativo" (PNUD, 2010: 16). Se basa en un indicador social estadístico compuesto por tres variables: 1) vida larga (medida según la esperanza de vida al nacer); 2) educación (medida por la tasa de alfabetización de adultos y la tasa bruta combinada de matrícula en educación primaria, secundaria y superior, así como los años de duración de la educación), y 3) nivel de vida (medido por el PIB per cápita PPA en dólares).

El PNUD clasifica los países en tres grandes grupos: con desarrollo humano alto (IDH $\geq 0,8)$; con desarrollo humano medio $(0.5 \leq \mathrm{IDH}<$ 0.8 ); y con desarrollo humano bajo (IDH $<0.5$ ).

Raworth y Steward (2002: 141) 3 hicieron una revisión de las aplicaciones del IDH, junto con un compendio de las principales críticas imputables al mismo, entre las que destacan la falta de inclusión en su cálculo de dimensiones relevantes para el desarrollo, algunas de las cuales incluimos en este trabajo, como las políticas medioambientales o la igualdad de género (Brand, 2001; Rodenberg, 1997).

Asimismo, desde un punto de vista metodológico, una de las principales limitaciones del IDH es la redundancia en el cálculo del indicador, junto con la ponderación arbitraria de sus componentes (Rodas, 2000), lo que pretendemos, asimismo, solucionar con la utilización del método $\mathrm{DP}_{2}$, en el cual incluimos también a países para los que no se ha estimado el IDH para el año en cuestión, como en el caso de Somalia.

\section{Un indicador social sintético de bienestar: el de distancia $P_{2}$ de Pena Trapero}

La aplicación original del indicador con base en la distancia $\mathrm{P}_{2}$ va dirigida al campo de la medición del bienestar social, el desarrollo sostenible, etc., entre distintas regiones o países (Sánchez y Rodríguez, 2003; López, 2003; Castro, 2004; Escobar, 2006; Somarriba y Pena, 2008, 2009; Zarzosa, 2009).

Se trata de un indicador global que proporciona una visión conjunta de la situación de los individuos de una colectividad en relación con el concepto a estudiar. Una gran ventaja de este método es que resuelve la cuestión de agregación de variables expresadas en distintas medidas, la ponderación arbitraria y la duplicidad de información (Zarzosa, 1996).

${ }^{3}$ A este respecto, también puede verse el trabajo de McNeill (2007). 


\subsection{Descripción del modelo estadístico}

Un indicador sintético representa una función matemática de variables de la forma $\mathrm{I}=\mathrm{F}\left(\mathrm{X}_{1}, \mathrm{X}_{2}, \ldots . \mathrm{X}_{\mathrm{n}}\right)$, donde $n$ es el número de indicadores parciales que incorporan información sobre el bienestar, en nuestro caso. Así, el porcentaje de mujeres en el parlamento sería un indicador parcial de la igualdad de género en un territorio en particular, y de su grado de bienestar, en general, que se recoge en I.

Por tanto, si $m$ es el número de países, en una matriz $X$ de observaciones, de orden $m \times n$, el componente $\mathrm{X}_{\mathrm{ij}}$ reflejará la situación de la variable $i$ en el país $j$.

El indicador $\mathrm{DP}_{2}$ de Pena Trapero (1977), nos dará las distancias de cada país respecto a un país ficticio de referencia, en el cual se obtendría el valor mínimo en el conjunto de los indicadores sociales considerados. Así, $m$ es el número de países; $n$ el número de variables; $\mathrm{X}_{\mathrm{ij}}$ el valor de la variable $i$ en el país $j$; $\sigma_{\mathrm{i}}$ es la desviación típica de la variable $i$; $\mathrm{y}_{\mathrm{i}, \mathrm{i}-1, \ldots, 1}^{2}$ es el coeficiente de determinación, que mide la parte de la varianza de cada variable explicada por la regresión lineal realizada sobre las variables precedentes. Este coeficiente es un número abstracto independiente de las unidades de medida en que estén expresadas las distintas variables.

Atendiendo a una de las principales ventajas comentadas del índice $\mathrm{DP}_{2}$, el factor corrector $\left(1-\mathrm{R}_{\left.\mathrm{i},{ }_{\mathrm{i}-1}, \ldots_{1}\right)}\right)$, que refleja la parte de la varianza de $\mathrm{X}_{\mathrm{i}}$ no explicada por $\mathrm{X}_{\mathrm{i}-1}, \mathrm{X}_{\mathrm{i}-2}, \ldots \mathrm{X}_{1}$, no permite la duplicidad de información, ya que descarta de las variables la información contenida en las anteriores. Es decir, se ponderan las diferencias en la variable $i$-ésima entre un país y el de referencia, por la proporción de información útil (no proporcionada por el resto del indicador) que aporta esa variable.

$\mathrm{El} \mathrm{DP}{ }_{2}$ se construye como:

$$
D P 2_{i}=\sum_{j=1}^{m} \frac{d_{i j}}{\sigma_{j}} 1\left(1-R_{j ; 1, \ldots, j-2, j-1}^{2}\right)
$$

con $j=1, \ldots . m$; y, por definición, $R_{1}^{2}=0$, donde, $\mathrm{d}_{\mathrm{ij}}$ es la diferencia entre el valor que toma la variable $i$-ésima en el país $j$ y el mínimo de la variable en el escenario teórico menos deseable, tomado como marco de referencia.

Como se pretende medir el grado de bienestar en distintos territorios para poder realizar comparaciones, el $\mathrm{DP}_{2}$ contempla las diferencias en bienestar social, ya que en cada una de las variables se considera como base de referencia el valor correspondiente al país que registra el menor valor.

Por tanto, un mayor valor de $\mathrm{DP}_{2}$ muestra más bienestar, al suponer una mayor distancia respecto al marco teórico menos deseado, en el que se 
alcanzaría un importe mínimo en el conjunto de los indicadores parciales considerados. $^{4}$

\subsection{Propiedades matemáticas del indice $\mathrm{DP}_{2}$}

Un indicador sintético debe cumplir una serie de propiedades matemáticas para poder proporcionar una buena medida o estimación del objeto a medir. El indicador sintético $\mathrm{DP}_{2}$ verifica esas propiedades (Pena Trapero, 1977: 49 y ss.; Zarzosa, 1996; Escobar, 2006: 80), concretamente:

1) Existencia y determinación. La función matemática que define el indicador sintético debe ser tal que exista y tenga solución para todos los valores del índice en cada unidad de observación.

2) Monotonía. El índice debe responder favorablemente a una modificación positiva de los componentes y negativamente a una en signo contrario. En la práctica, esta condición exige que en el modelo se introduzca la información con el signo con que se espera estén relacionados los indicadores simples y el objetivo a medir.

3) Unicidad. El índice debe representar un único valor, para una situación determinada.

4) Invarianza. Como consecuencia de la propiedad anterior, el índice debe ser invariante respecto a un cambio de origen y/o escala de medición de los indicadores simples.

5) Homogeneidad. La función matemática que defina el índice debe ser homogénea de grado uno. Este hecho asegura que si los indicadores simples aumentan o disminuyen, en igual proporción lo hace el indicador sintético.

6) Transitividad. En el sentido que teniendo tres valores numéricos del indicador sintético, cuando el primero es mayor que el segundo, y el segundo, a su vez, mayor que el tercero, se debe verificar que el primero es mayor que el tercero.

7) Exhaustividad. El índice debe ser tal que aproveche al máximo y de forma útil la información suministrada por cada uno de los indicadores simples.

8) Aditividad. El indicador sintético de distancia definido para la comparación entre dos países $(r, k)$ debe ser tal que, la diferencia obtenida entre ellos directamente por el indicador de distancia, sea igual a la que se obtendría al comparar los indicadores sintéticos de cada país. No obstante, la $\mathrm{P}_{2}$ no verifica estrictamente la pro-

\footnotetext{
${ }^{4}$ Para una descripción exhaustiva de la metodología del indicador $\mathrm{DP}_{2}$, véase Pena (1977) y Zarzosa $(1994,1996)$.
} 
piedad de aditividad, sin embargo, Zarzosa $(1992,1996)$ demuestra que la relación que existe entre ambos es:

$$
\left|\mathrm{DP}_{2}(\mathrm{r})-\mathrm{DP}_{2}(\mathrm{k})\right| \leq \mathrm{DP}_{2}(\mathrm{r}, \mathrm{k}) \text {. }
$$

9) Invarianza respecto a la base de referencia. El indicador sintético de distancia definido para la comparación entre los países debe ser invariante respecto a la base de referencia que se tome para cada país, con tal de que sea la misma para todos ellos.

10) Conformidad. Establece que el propio indicador debe determinar que el rango de los indicadores parciales es correcto (Zarzosa, 1996).

11) Neutralidad. El peso de cada indicador simple vendría dado por la información útil que tuviera cada uno, de acuerdo con la varianza explicada del índice general.

De este modo, con el $\mathrm{DP}_{2}$ se aplica un método no aleatorio, más consistente que otros procedimientos con objetivos similares, como el de componentes principales, que permiten también construir indicadores sintéticos de bienestar, como se recoge en García Lautre (1998), si bien el método de componentes principales presenta algunos problemas en el cumplimiento de algunas de las condiciones matemáticas enunciadas antes, como la invarianza, la homogeneidad y, especialmente, la neutralidad (Ram, 1982; Pena, 2009).

\section{El indicador sintético de bienestar social en los PMA de África para 2006}

\subsection{Planteamiento}

Como se mencionó, se pretende elaborar un indicador sintético de bienestar social que permita analizar las disparidades existentes en 2006 entre 31 países PMA de África, de acuerdo con el mayor o menor cumplimiento de los Objetivos del Milenio (ODM), que representan una asociación global que surgió de los compromisos y metas establecidas en las cumbres mundiales de los ańos noventa. Así, fijados en el año 2000, los oDM son ocho objetivos globales para mejorar el destino de la humanidad en el nuevo siglo, que constituyen, en la actualidad, la principal agenda internacional del desarrollo que los 191 países miembros de las Naciones Unidas acordaron conseguir para el ańo 2015 (onU, 2009; cuadro 1), con referencia a la situación mundial de 1990 . En particular, se refieren a la erradicación de la pobreza, la educación primaria universal, la igualdad entre los géneros, la mortalidad infantil, la salud materna, el avance del 
SIDA y otras enfermedades, el sustento del medio ambiente y fomentar una asociación mundial para el desarrollo.

La estimación del $\mathrm{DP}_{2}$ requiere de varios pasos en su construcción, así como diversas reordenaciones matriciales en cada etapa del procedimiento iterativo. En un primer momento del procedimiento, hay que elegir las variables teniendo en cuenta que cuanto mayor sea la cantidad de información que aporta un indicador no contenida en la información global de los anteriores ya incorporados al indicador sintético, mejor será el indicador parcial.

En nuestro trabajo se empleó la detallada y fiable información estadística aportada por el informe, en su versión impresa, sobre los PMA (UNCTAD, 2008).

\subsection{Selección de variables o indicadores parciales}

De un número inicial de variables bastante elevado se seleccionaron 23, que distribuimos entre los ocho Objetivos del Milenio (onU, 2009), como se observa en el cuadro 1, con los últimos datos disponibles. Este paso se ha desarrollado en dos etapas: determinación de las variables necesarias e identificación de las variables efectivamente disponibles (Trapé, 2000).

El año de análisis es 2006, pero de aquellas variables para las que no se disponía de información a esa fecha, se tomó como alternativa el año más próximo. ${ }^{5}$

Finalmente, hay que tener en cuenta que las variables que registran una relación negativa con el bienestar social, es decir, aquéllas cuyos aumentos pueden ir unidos a disminuciones del bienestar, se disponen en la matriz de observaciones $\mathrm{X}$ con signo negativo, de acuerdo con la propiedad de monotonía del indicador $\mathrm{DP}_{2}$ comentada. Concretamente, las variables con signo negativo serían las vinculadas a los objetivos 4a, 5a, 6a, 7 a y $8 d$ (cuadro 1 ).

\section{Resultados del indicador sintético $\mathrm{DP}_{2}$ de bienestar social en los PMA de África, 2006}

En esta parte del trabajo se pretende realizar como aportación propia una aplicación del indicador sintético distancia- $\mathrm{P}_{2}$ al caso de los PMA de África, tomando como referencia los Objetivos de Desarrollo del Milenio.

\footnotetext{
${ }^{5}$ Esto ha sucedido en las variables: porcentaje de la superficie terrestre cubierta por bosques; emisión de dióxido de carbono; esperanza media de vida al nacer; oferta total de alimentos; consumo de electricidad e indicadores de educación, cuya información disponible es de 2005; en tanto que el porcentaje de la población que utiliza instalaciones sanitarias adecuadas, y de población que usa fuentes mejorables de agua potable y la densidad de las carreteras $\left(\mathrm{km} / 1,000 \mathrm{~km}^{2}\right)$ se emplea la existente en 2004 .
} 


\section{Cuadro 1 \\ Variables de bienestar social por Objetivos de Desarrollo del Milenio de la ONU}

Objetivo 1. Erradicar la pobreza extrema y el hambre

a) PIB real per cápita

b) Tasa de actividad

c) Oferta total de alimentos ( $\mathrm{k} /$ clas diarias) per cápita

d) Consumo de electricidad (Kv/hora) per cápita

Objetivo 2. Educación universal

a) Tasa neta de matrícula escolar (total población) en educación primaria

b) Tasa de alfabetización de adultos

Objetivo 3. Igualdad entre géneros

a) Tasa neta de matrícula escolar (mujer) en educación primaria

b) Fuerza de trabajo de la mujer (\%)

c) Mujeres en el Parlamento (\%)

Objetivo 4. Reducir la mortalidad de los niños

a) Tasa de mortalidad infantil de niños menores de un año

b) Porcentaje de niños de un ańo de edad inmunizado contra DPT3

c) Esperanza media de vida al nacer

Objetivo 5. Mejorar la salud materna

a) Mortalidad materna por 100,000 nacidos vivos

b) Porcentaje sobre la media del conjunto de los PMA de partos atendidos por personal especializado

Objetivo 6. Lucha contra el SIDA y otras enfermedades infecciosas

a) Prevalencia estimada (\%) de VIH en adultos

Objetivo 7. Sostenibilidad del medio ambiente

a) Emisión de $\mathrm{CO}_{2}(\mathrm{t} / \mathrm{m})$ per cápita

b) Porcentaje de superficie terrestre cubierta por bosques

c) Proporción de la población que utiliza fuentes mejoradas de agua potable

d) Porcentaje de la población que utiliza instalaciones sanitarias adecuadas

Objetivo 8. Fomentar una alianza mundial para el desarrollo

a) Participación exportaciones PMA en mercados de las economías desarrolladas

b) Red de carreteras. Densidad $\left(\mathrm{km} / 1,000 \mathrm{~km}^{2}\right)$

c) Número de líneas de teléfono por 1,000 habitantes

d) Ratio deuda externa/PIB

Fuente: Elaboración propia con base en oNU (varios años).

\subsection{Resultados del indicador $D P_{2}$. Clasificación por países}

Para evaluar correctamente los resultados del $\mathrm{DP}_{2}$, como se indicó, hay que tomar en consideración que se ha utilizado una base de referencia formada por la peor situación teórica; es decir, un país ficticio, que arrojaría los menores valores observados de las variables utilizadas. De este modo, un mayor valor de $\mathrm{DP}_{2}$ de un país refleja una mejoría en cuanto a la situación de bienestar, porque supone una mayor distancia respecto al escenario teórico menos deseado. 
Por países, mediante las estimaciones obtenidas, se puede observar como Djiboutí era el que presentaba la mejor situación real de bienestar en 2006, con una distancia a la base de referencia igual a 30.25 (cuadro 2). Le seguían Zambia y Senegal, del África austral y del oeste, respectivamente, que presentaban también valores relativos elevados en el conjunto de los indicadores sociales considerados.

Asimismo, de acuerdo con nuestra clasificación, destaca la posición privilegiada de países pertenecientes al este y oeste África, es el caso de Guinea Ecuatorial (26.73) y Gambia (26.79), que se encontraban por encima de la distancia media (22.95). Níger, por el contrario, registró la situación peor entre el conjunto de los países, con una distancia a la base de referencia de 11.89 .

Este hecho significa que la máxima distancia interpaíses, es decir, entre la máxima y mínima valoración obtenida, fue de 18.36 , lo que muestra que las disparidades entre los PMA en el nivel de cumplimiento de los Objetivos de Desarrollo del Milenio eran elevadas en el año de referencia, conclusión que coincide, en general, con la obtenida por el PNUD (2010).

Por otra parte, como se observa en el cuadro 2, se puede afirmar que apenas hay distancia entre los dos países con una situación más negativa, Chad y Níger, en tanto que sí la hay, en mayor medida, entre los dos países con un resultado más positivo, Djiboutí y Zambia.

En la clasificación de países, en función del IDH en 2006 (cuadro 2), las tres primeras posiciones entre los PMA de África corresponden a Guinea Ecuatorial, Mauritania y Lesotho, con un nivel de desarrollo medio, Djiboutí ocupa el puesto seis. Sobre este punto hay que recordar que el IDH no considera entre sus variables a las encuadradas en nuestro trabajo en el Objetivo 3 del Milenio: igualdad de género; en el Objetivo 7: sostenibilidad del medio ambiente; o en el Objetivo 8: fomentar una alianza mundial para el desarrollo. En este sentido, la clasificación de países obtenida con el $\mathrm{DP}_{2}$ no coincide, en general, con el ranking del IDH (cuadro 2).

Asimismo, según el $\mathrm{DP}_{2}$, países del oeste y del Cuerno de África, como Níger, Chad, Etiopía, Somalia y Sierra Leona, respectivamente, que representan casi la cuarta parte de la población total de los PMA, registraban los niveles más bajos, en línea con la clasificación del IDH. Estos países se sitúan relativamente cerca de la situación teórica menos deseable (cuadro 2 ), con una distancia por debajo de 16, ya que en la mayoría de los indicadores sociales analizados figuran con valores mínimos.

En esta misma dirección, es significativo también el lugar poco privilegiado en el que figuran otros tres países del oeste, como Liberia, Guinea Bissau y Malí, con valores inferiores a la media y próximos al umbral mínimo de referencia. 


\section{Cuadro 2}

Indicadores sintéticos de bienestar social en los PMA africanos, 2006 Orden países $\mathrm{DP}_{2}$ relativa e índice de desarrollo humano de la $\mathrm{ONU}$

\begin{tabular}{|c|c|c|c|c|}
\hline Pais & $\begin{array}{c}\text { Indicador } \\
\mathrm{DP}_{2}\end{array}$ & $I D H$ & $\begin{array}{c}\text { \% sobre el total } \\
\text { Población } \\
\text { PMA África }\end{array}$ & $\begin{array}{c}\text { Delimitación } \\
\text { geográfica en el } \\
\text { continente }\end{array}$ \\
\hline Djiboutí & 30.25 & 0.52 & 0.17 & Cuerno de África \\
\hline Zambia & 28.76 & 0.43 & 2.43 & Austral \\
\hline Senegal & 28.54 & 0.5 & 2.51 & Oeste \\
\hline Gambia & 26.79 & 0.5 & 0.35 & Oeste \\
\hline Guinea Ecuatorial & 26.73 & 0.64 & 0.10 & Centro \\
\hline Malawi & 26.56 & 0.44 & 2.83 & Austral \\
\hline Lesotho & 26.19 & 0.55 & 0.42 & Austral \\
\hline Uganda & 26.06 & 0.49 & 6.21 & Este \\
\hline Rwanda & 25.81 & 0.45 & 1.97 & Este \\
\hline Mozambique & 25.18 & 0.39 & 4.36 & Austral \\
\hline \multicolumn{5}{|l|}{ República Unida } \\
\hline de Tanzania & 25.11 & 0.47 & 8.21 & Este \\
\hline Isla de Madagascar & 24.69 & 0.53 & 3.99 & Océano Índico \\
\hline Mauritania & 24.35 & 0.56 & 0.62 & Oeste \\
\hline Sudán & 23.95 & 0.53 & 7.83 & Cuerno de África \\
\hline Benin & 23.2 & 0.44 & 1.83 & Oeste \\
\hline Togo & 22.68 & 0.48 & 1.33 & Oeste \\
\hline Eritrea & 22.29 & 0.44 & 0.98 & Cuerno de África \\
\hline Burundi & 22.01 & 0.41 & 1.70 & Este \\
\hline Angola & 21.29 & 0.45 & 3.45 & Austral \\
\hline Guinea & 20.56 & 0.46 & 1.91 & Oeste \\
\hline Burkina Faso & 19.64 & 0.37 & 2.99 & Oeste \\
\hline \multicolumn{5}{|l|}{ República } \\
\hline Centroafricana & 19.17 & 0.38 & 0.89 & Centro \\
\hline \multicolumn{5}{|l|}{ República } \\
\hline Democrática & & & & \\
\hline del Congo & 18.99 & 0.41 & 12.59 & Centro \\
\hline Malí & 18.64 & 0.38 & 2.49 & Oeste \\
\hline Guinea-Bissau & 18.4 & 0.37 & 0.33 & Oeste \\
\hline Liberia & 17.47 & 0.36 & 0.75 & Oeste \\
\hline Sierra Leona & 15.78 & 0.32 & 1.18 & Oeste \\
\hline Etiopía & 15.01 & 0.39 & 16.83 & Cuerno de África \\
\hline Somalia & 12.3 & n.d.* & 1.75 & Cuerno de África \\
\hline Chad & 12.13 & 0.39 & 2.18 & Oeste \\
\hline Níger & 11.89 & 0.37 & 2.85 & Oeste \\
\hline
\end{tabular}

* No disponible.

Fuente: Elaboración propia con base en UNCTAD (2008), ONU (varios años). 
Finalmente, hay que mencionar que Guinea Ecuatorial es el único país de África Central que alcanzó una posición relativamente alta en la lista, con valores similares a Malawi y Gambia (cuadro 2).

\subsection{Ordenación de los indicadores parciales}

En el cuadro 3 se recoge la ordenación de los indicadores parciales determinada mediante el cálculo iterativo de la $\mathrm{DP}_{2}$, así como el factor corrector de cada uno de ellos. Se muestra el orden de entrada de cada variable y el peso que ha tenido cada una en el indicador final. El número de orden en el que presentamos cada variable corresponde con el orden de entrada en el indicador. Por ejemplo, la variable 1 sería el porcentaje de la población que utiliza instalaciones sanitarias adecuadas.

En función de los resultados arrojados por el $\mathrm{DP}_{2}$, se corrobora la idoneidad de la aplicación de este indicador sintético de cara a nuestros objetivos iniciales, ya que se detectan altas correlaciones entre diferentes indicadores parciales, lo que originaría excesiva redundancia si no se suprimiese, como hace la $\mathrm{DP}_{2}$, la información repetida. De este modo, la variable 3 (tasa de alfabetización de adultos), por ejemplo, resulta relevante en la explicación del nivel de bienestar social de los países, ocupando la posición cuarta, según su factor de corrección de 0.77 (cuadro 3). Este dato significa que únicamente $23 \%$ de su variabilidad fue explicada por las dos primeras variables, recogiendo, por tanto, $77 \%$ de la información nueva o útil.

En función del orden relativo en el que se conforman las variables, se puede deducir que las incluidas en los objetivos 7 y 5 ejercen de forma clara la mayor influencia en la determinación del índice $\mathrm{DP}_{2}$ calculado. En particular, los indicadores parciales 1 (porcentaje de la población que utiliza instalaciones sanitarias adecuadas) y 2 (mortalidad materna por 100,000 nacidos vivos) ocupan las dos primeras posiciones en la ordenación, con un elevado peso en el resultado final.

Le siguen a poca distancia las variables incorporadas en los objetivos 2 (educación universal) y 3 (igualdad de la mujer). Estas variables también se encuentran muy vinculadas con el bienestar social de los PMA, destacando la repercusión de la variable 3 (tasa de alfabetización de adultos) y la 5 (tasa neta de matricula escolar de la mujer en educación primaria).

Asimismo, sería reseñable la importancia del indicador 4 (porcentaje de la población que utiliza fuentes mejoradas de agua potable), encuadrado en el Objetivo 7 del Milenio, en la clasificación de los pMa de África. Además, presenta un factor de corrección de 0.67 , por lo que aporta un grado elevado de información útil (nueva). 
Cuadro 3

Clasificación de los indicadores parciales por orden de entrada en la $\mathrm{DP}_{2}$ Orden de variables, factor corrector y coeficiente de determinación

\begin{tabular}{|c|c|c|c|}
\hline Variable & $\begin{array}{l}\text { Objetivo del } \\
\text { Milenio }\end{array}$ & $\begin{array}{c}\text { Pesos } \\
\text { (factor de } \\
\text { corrección) }\end{array}$ & $\begin{array}{l}|r| \\
(\%)\end{array}$ \\
\hline $\begin{array}{l}\text { Población que utiliza instalaciones } \\
\text { sanitarias adecuadas } \\
\text { Mortalidad materna por } 100,000\end{array}$ & 7 & 1 & 54.82 \\
\hline nacidos vivos & 5 & 0.89 & 34.28 \\
\hline Tasa de alfabetización de adultos & 2 & 0.77 & 42.35 \\
\hline $\begin{array}{l}\text { Población que utiliza fuentes mejoradas } \\
\text { de agua potable }\end{array}$ & 7 & 0.76 & 34.42 \\
\hline $\begin{array}{l}\text { Tasa neta de matrícula escolar (mujer) } \\
\text { en educación primaria }\end{array}$ & 3 & 0.71 & 27.79 \\
\hline $\begin{array}{l}\text { Porcentaje sobre la media del conjunto } \\
\text { de los PMA de partos atendidos por personal } \\
\text { especializado } \\
\text { Número de líneas de teléfono por } 1,000\end{array}$ & 5 & 0.67 & 26.82 \\
\hline habitantes & 8 & 0.65 & 27.47 \\
\hline $\begin{array}{l}\text { Mujeres en el Parlamento } \\
\text { Niños de un año de edad inmunizado contra }\end{array}$ & 3 & 0.57 & 22.90 \\
\hline $\begin{array}{l}\text { DPT3 } \\
\text { Oferta total de alimentos (k/clas diarias) }\end{array}$ & 4 & 0.56 & 13.51 \\
\hline $\begin{array}{l}\text { per cápita } \\
\text { Tasa de mortalidad infantil de los menores }\end{array}$ & 1 & 0.49 & 19.75 \\
\hline de un año & 4 & 0.41 & 12.60 \\
\hline Red de carreteras. Densidad km/1,000 km² & 8 & 0.4 & 25.69 \\
\hline $\begin{array}{l}\text { Emisión de } \mathrm{CO}_{2}(\mathrm{t} / \mathrm{m}) \text { per cápita } \\
\text { Proporción de superficie terrestre cubierta }\end{array}$ & 7 & 0.38 & 11.88 \\
\hline por bosques & 7 & 0.35 & 2.93 \\
\hline $\begin{array}{l}\text { Participación de las exportaciones de los PMA } \\
\text { en los mercados de las economías desarrolladas }\end{array}$ & 8 & 0.35 & 2.66 \\
\hline PIB per cápita & 1 & 0.31 & 3.59 \\
\hline Esperanza media de vida al nacer & 4 & 0.28 & 11.67 \\
\hline Ratio deuda externa/PIB & 8 & 0.21 & 4.65 \\
\hline Tasa de actividad & 1 & 0.2 & 3.29 \\
\hline Emisión de $\mathrm{CO}_{2}(\mathrm{t} / \mathrm{m})$ per cápita & 7 & 0.13 & 6.24 \\
\hline Fuerza de trabajo de la mujer & 3 & 0.13 & 1.98 \\
\hline Prevalencia estimada de viH en adultos & 6 & 0.08 & 11.08 \\
\hline $\begin{array}{l}\text { Tasa neta de matrícula escolar (total } \\
\text { población) en educación primaria }\end{array}$ & 3 & 0.02 & 20.50 \\
\hline
\end{tabular}

Fuente: Elaboración propia con base en UNCTAD (2008), onu (varios años). 
En el marco del Objetivo 8, fomentar una alianza mundial para el desarrollo, hay que resaltar la posición alta en la clasificación de la variable 7: número de líneas de teléfono por 1,000 habitantes. Además, aunque la variable 15 (participación de las exportaciones de los PMA en los mercados de las economías desarrolladas) figura en un rango bajo, tiene un importante grado de información no incluida en las anteriores. Singularmente, este indicador retiene $35 \%$ de información no duplicada ni incorporada en los 16 anteriores, de ahí el interés de incorporarlo también al $\mathrm{DP}_{2}$.

En este sentido, y dadas las dificultades para encontrar estadísticas actualizadas relativas al Objetivo 5 (mejora de la salud materna), cabe subrayar la elevada repercusión de la variable 6 (porcentaje sobre la media del conjunto de los PMA de partos atendidos por personal especializado) en la construcción del $\mathrm{DP}_{2}$. Además, presenta un factor de corrección de $67 \%$, lo que conduce a pensar que su grado de relevancia no es nada despreciable.

Sobre este punto se puede destacar la importancia que hay que otorgarle a los avances en el campo de la salud y de la educación de los PMA de cara a incrementar el bienestar social de estos países, pero también a otras dimensiones del desarrollo como la igualdad entre géneros o la sostenibilidad del medio ambiente, que incorporamos en nuestro trabajo.

Por el contrario, los componentes más vinculados al empleo, como el 19 (tasa de actividad), no parecen ser los más relacionados con el cumplimiento de los Objetivos del Milenio de la onu, en la esfera del desarrollo de estos países (cuadro 1), probablemente porque su información está incluida en otras variables de su grupo o de otros componentes.

Por su parte, la variable 25 (PIB per cápita) presenta un factor de corrección de 0.31 , reteniendo $31 \%$ de información nueva, de modo que parte de su información está contenida en otras variables que le anteceden de su componente (erradicar la pobreza extrema y el hambre) o de otros grupos (cuadro 3), es decir, cuando se ha considerado en el procedimiento del $\mathrm{DP}_{2}$ esta variable, se ha eliminado la información redundante.

Por último, hay que resaltar que la única variable que aporta la totalidad de su información (nueva y útil), o lo que es lo mismo, el único indicador parcial que no se corrige, es el 1 (porcentaje de la población que utiliza instalaciones sanitarias adecuadas) del Objetivo 7: sostenibilidad del medio ambiente.

\section{Conclusiones}

Debido a que el bienestar social engloba aspectos materiales e inmateriales de difícil cuantificación, su medición no es sencilla. No obstante, en este trabajo se realizó como aportación propia una aproximación a la 
medición del bienestar social de los PMA de África en 2006, con la construcción de un indicador global de cumplimiento de los ocho Objetivos del Milenio de Naciones Unidas.

Para ello se optó por el método multidimensional $\mathrm{DP}_{2}$, que soluciona mediante un método científico las principales limitaciones de un indicador sintético, en particular, el carácter desagregado de las medidas, la falta de neutralidad en la ponderación de los indicadores parciales y la redundancia de la información.

En nuestro trabajo, a partir de 23 variables, seleccionadas previamente y referidas a los Objetivos del Milenio de la ONU, se calculó el indicador en 31 PMA de África, distribuidos en cinco agrupaciones geográficas del continente (centro, este, oeste, Cuerno de África y austral), con los siguientes resultados:

1) En principio, la mayoría de las variables analizadas aportarían información relevante para la determinación y la medición cardinal del bienestar social de los pMa de África.

2) Las variables de los objetivos 7 (sostenibilidad del medio ambiente), 5 (mejorar la salud materna) y 2 (educación universal) parecen mostrar la mayor correlación con el indicador sintético de bienes$\operatorname{tar} \mathrm{DP}_{2}$, con un gran peso en la clasificación final de los países, de acuerdo con el orden en que se obtienen los indicadores parciales.

3) Djiboutí, situado en el Cuerno de África, es el país que alcanzó mayor grado de bienestar social real en 2006, con una situación privilegiada en el conjunto de los PMA. Le siguen Zambia de la parte austral y, muy próximos entre sí, dos países del oeste, Senegal y Gambia, con una población relativamente pequeña. Estos países se podrían considerar los de mayor cumplimiento de los Objetivos del Milenio hasta 2006.

4) Los países más poblados, tanto del oeste, Chad y Níger, como del Cuerno de África, Etiopía y Somalia, serían los que se encuentran más cerca de la situación teórica menos deseable, es decir, los que presentaban los valores más bajos en las variables incorporadas a nuestro indicador global. Esta situación afectaba a casi la cuarta parte de los habitantes totales de los pMA en 2006.

5) Los valores del indicador $\mathrm{DP}_{2}$ evidencian un cierto nivel de disparidad en el cumplimiento de los Objetivos del Milenio de los PMA de África en el periodo considerado. Este hecho se podría tener más en cuenta en la programación de ayuda de los organismos internacionales, intensificando los esfuerzos a favor del desarrollo en aquellos territorios que presentan valores más bajos en los indicadores sociales estudiados. 
6) Algunas de las variables que registraron mayores diferencias en sus magnitudes por países no se suelen incluir en otros índices elaborados con objetivos similares, concretamente, el ratio deuda externa/PIB, la participación de las exportaciones de los PMA en los mercados de las economías desarrolladas; y la red de carreteras, densidad $\mathrm{km} / 1,000 \mathrm{~km}^{2}$, por lo que consideramos conveniente incluir este tipo de variables en las medidas sintéticas del bienestar.

Para finalizar, únicamente nos queda reiterar nuestra intención de que este trabajo constituya una aportación en el intento de aproximarse a la medición y la mejora del bienestar social, en este caso, de los PMA de África. Nuestro objetivo es establecer prioridades sobre aquellos aspectos que son importantes para conseguir los Objetivos de Desarrollo del Milenio en este grupo de países.

Los resultados obtenidos en cuanto a la elevada importancia relativa de variables como la educación y la salud en el bienestar social de los PMA de África, tienen un alto grado de coincidencia con los del IDH y los informes del PNUD. Sin embargo, en nuestro trabajo también se pone de manifiesto la repercusión considerable en el cumplimiento de estas metas con respecto a otras dimensiones consideradas, como la igualdad de género, la sostenibilidad del medio ambiente o las relaciones exteriores de estos territorios, destacando variables como el porcentaje de mujeres en el Parlamento o el número de líneas de teléfono por 1,000 habitantes, pues proporcionan una parte de información no redundante en la construcción del indicador $\mathrm{DP}_{2}$.

En este sentido, los países pobres son especialmente vulnerables a las crisis mundiales, y la situación de inestabilidad económica actual puede poner en peligro los progresos logrados en los odm. En particular, los relacionados con el género enfrentan riesgos adicionales, ya que los datos disponibles de crisis pasadas indican que las mujeres, en general, son más vulnerables a sus efectos. Por esta razón, se hace más necesario tener en cuenta los aspectos relativos a la igualdad de género en las medidas de respuesta a escala internacional y nacional, e incorporar en los diferentes índices de bienestar variables como la tasa neta de matrícula escolar (mujer) en educación primaria o la participación de la mujer en el Parlamento, entre otras.

Asimismo, aunque se han hecho progresos con respecto al objetivo de mejorar el acceso a agua potable y a instalaciones sanitarias adecuadas, también es probable que haya graves problemas en los próximos años para seguir avanzando en esta dirección, aspecto muy relevante para mejorar el bienestar futuro de los PMA de África, según se desprende de nuestras estimaciones. 
Por último, además de los componentes anteriores, en el sector de salud se deberá prestar especial atención a variables como la evolución de la mortalidad materna y el porcentaje de partos atendidos por personal especializado, o el porcentaje de niños de un año de edad inmunizados contra enfermedades infecciosas, con un alto peso específico en la determinación final del indicador de bienestar $\mathrm{DP}_{2}$, para seguir avanzando en el cumplimiento de los ODM en estos países, en el horizonte temporal de 2015.

\section{Bibliografía}

Banco Mundial (2001), Informe sobre el desarrollo mundial 2000-2001. Lucha contra la pobreza, Banco Mundial, Washington.

Brand, Peter (2001), "La construcción ambiental del bienestar humano", Economia, Sociedad y Territorio, 3 (9), El Colegio Mexiquense, Zinacantepec, pp. 1-24.

Castro, Juan (2004), Indicadores de desarrollo sostenible urbano. Una aplicación para Andalucía, Instituto de Estadística de AndalucíaConsejería de Economía y Hacienda, Sevilla.

Consejo de Europa (2003), Conclusiones del Consejo sobre indicadores estructurales, documento 15.875/03, Consejo de la Unión Europea, Bruselas.

Chasco, Coro e Inve Hernández (2003), Medición del bienestar social microterritorial, Instituto L. R. Klein-Universidad Autónoma de Madrid, Madrid.

Edgeworth, Francis (1897), “The Pure Theory of Taxation”, The Economic Journal, 7 (25), Royal Economic Society, Londres, pp. 46-70.

Escobar, Luis (2006), "Indicadores sintéticos de calidad ambiental: un modelo general para grandes zonas urbanas", EURE, 32 (96), Santiago de Chile, pp. 73-98.

García, José y Pedro Puig (1980), La calidad de vida en España. Hacia un estudio de indicadores sociales, Moneda y Crédito, Madrid. 
García-Lautre, Ignacio (1998), "Una propuesta metodológica para la ordenación de las infraestructuras regionales", Estudios Regionales, 51, Universidad de Andalucía, Sevilla, pp. 145-170.

Hicks, John (1939), "The Foundations of Welfare Economics”, The Economic Journal, 49, Royal Economic Society, Londres, pp. 696-712.

INE (Instituto Nacional de Estadística) (1999), Indicadores sociales, INE, Madrid.

Jasek-Rysdahl, Kelvin (2001), “Applying Sen's Capabilities Framework to Neighborhoods: Using Local Asset Maps to Deepen our Understanding of Well-being", Review of Social Economy, 49 (3), Routledge, Taylor and Francis, Nueva York, pp. 312-329.

López, María (2003), "Bienestar socioeconómico de los municipios gallegos: una aproximación a su medida”, Revista Galega de Economía, 12 (2), Universidad de Santiago de Compostela, Galicia, pp. $1-24$.

McNeill, Desmond (2007), "Human Development: The Power of the idea", Journal of Human Development, 8 (1), Routledge, Taylor and Francis, Nueva York, pp. 5-22.

ocde (Organización para la Cooperación y el Desarrollo Económico) (2002), Society at a Glance: OECD Social Indicator, OCDE, París.

ONU (Organización de las Naciones Unidas) (2009), Informe del Grupo de Tareas sobre el desfase en el logro de los objetivos de desarrollo del Milenio de 2009, onu, Nueva York.

Pareto, Vilfredo (1906), Manuel d'économie politique, V. Giard y E. Brière, París.

Pena, Bernardo (1977), Problemas de la medición del bienestar y conceptos afines (Una aplicación del caso español), INE, Madrid.

Pena, Bernardo (2009), "La medición del bienestar social: una revisión crítica", Estudios de Economía Aplicada, 2, Asepelt, Madrid, pp. 299-324.

Pigou, Arthur (1920), La economía del bienestar, Aguilar, Madrid. 
PNUD (Programa de las Naciones Unidas para el Desarrollo) (varios años), Informe sobre desarrollo humano, Oxford University Press, Nueva York.

PNUD (Programa de las Naciones Unidas para el Desarrollo) (2010), Balance sobre los Objetivos de Desarrollo del Milenio, reunión conjunta de las juntas ejecutivas del PNUD-UNFPA-UNICEF-PMA, 15-18 de enero.

Ram, Richard (1982), "International Inequality in the Basic Needs Indicators: A recent Perspective", World Development, 20, Office, Quebec, pp. 899-905.

Raworth, Kate y David Stewart (2002), Critiques of the Human Development Index: a review in Readings in Human Development, Oxford University Press, Oxford.

Rodenberg, Birte (1997), "Mujeres, medio ambiente y desarrollo: ¿tema de moda, no-tema, tema de la onu?, Economía, Sociedad y Territorio, 1 (2), El Colegio Mexiquense, Zinacantepec, pp. 407-426.

Sánchez, María Ángeles y Noelina Rodríguez (2003), “El bienestar social en los municipios andaluces en 1999", Revista Asturiana de Economía, 27, Asociación Asturiana de Estudios Económicos, Oviedo, pp. 99-119.

Rodas, Pablo (2000), La medición del desarrollo humano: una tarea interminable y polémica, CEPAL, México.

Sen, Amartya (1982), Choice, Welfare, and Measurement, The міт Press, Massachusetts.

Sen, Amartya (1991), "Welfare, Preference and Freedom", Journal of Econometrics, 3, Elsevier, Nueva York, pp. 15.

Sen, Amartya (1999), Development as Freedom. Oxford, Oxford University Press, Oxford.

Somarriba, Noelia y Bernardo Pena (2008), "Aproximación a un indicador regional y nacional de los Objetivos de Lisboa a partir de la medida de distancia $\mathrm{P}_{2}$ ”, Estudios Económicos de Desarrollo Inter- 
nacional, 8 (2), Universidad de Santiago de Compostela, Galicia, pp. 83-94.

Somarriba, Noelia y Bernardo Pena (2009), "Synthetic Indicators of Quality of Life in Europe", Social Indicators Research, 96, Springer, Dordrecht, pp. 115-133.

Trapé, Alejandro (2000), "Pautas cuantitativas y cualitativas para seleccionar instrumentos de política económica", documento de trabajo, Universidad Católica de Cuyo, San Juan.

unCTAD (Conferencia de las Naciones Unidas sobre Comercio y Desarrollo) (2008), Los paises menos adelantados. Informe de 2008, UNCTAD, Ginebra.

Zarzosa, Pilar (1992), "Aproximación a la medición del bienestar social, estudio de la idoneidad del indicador sintético 'distancia P2", tesis doctoral, Universidad de Valladolid, Valladolid.

Zarzosa, Pilar (1994), "El criterio de discriminación en la selección de indicadores de bienestar. Análisis del coeficiente de discriminación de Ivanovic", Estudios de Economía Aplicada, 2, Asepelt, Madrid, pp. 169-185.

Zarzosa, Pilar (1996), Aproximación a la medición bienestar social, Universidad de Valladolid, Valladolid.

Zarzosa, Pilar (2009), "Estimación de la pobreza en las comunidades autónomas españolas, mediante la distancia $\mathrm{DP}_{2}$ de Pena”, Estudios de Economía Aplicada, 27 (2), Asepelt, Madrid, pp. 397-415.

Recibido: 29 de enero de 2010. Reenviado: 22 de marzo de 2010. Aceptado: 14 de junio de 2010.

José Antonio Rodríguez-Martín. Es doctor en ciencias económicas y empresariales por la Universidad de Granada. Actualmente es profesor permanente en el Departamento de Economía Aplicada de la Universidad de Granada y miembro del Grupo de Investigación de la Junta de Andalucía (España): Economía Pública y Globalización. Sus líneas de investigación actuales son: presupuesto y hacienda pública de la Unión Europea, 
relaciones exteriores de la Unión Europea; bienestar social de los países menos adelantados (PMA). Entre sus últimas publicaciones destacan: "Análisis de las relaciones comerciales entre Iberoamérica y el Caribe y la Unión Europea (1992-2003), El Trimestre Económico, 73, Fondo de Cultura Económica, México, pp. 667-691 (2006); "Evolution of expenditure in the budget of the European Union", European Planning Studies, 15, Routledge, Taylor and Francis, Abingdon, pp. 1113-1126 (2007); "La atención a la integración laboral de las personas discapacitadas en la Unión Europea”, Revista de Fomento Social, 253, eteA, Córdoba, pp. 103-127 (2009); "Internet y la actividad exportadora de la industria española", Revista Comercio Exterior, 59 (10), Banco Nacional de Comercio Exterior, México, D.F. pp. 801-815 (2009); "Economía de la Unión Europea", Revista de Economía Institucional, 11 (20), Universidad Externado de Colombia, Bogotá, pp. 461-464 (2009). 